\title{
HELP apheresis in hypercholesterolemia and cardiovascular disease: efficacy and adverse events after 8,500 procedures
}

\author{
Frank van Buuren • Sven Kreickmann • \\ Dieter Horstkotte • Tanja Kottmann • \\ Klaus Peter Mellwig
}

Published online: 20 March 2012

(C) The Author(s) 2012. This article is published with open access at Springerlink.com

\begin{abstract}
Introduction Low density lipoprotein (LDL-C) apheresis is a last treatment option for hypercholesterolemic patients resistant to conservative lipid-lowering therapy. In a retrospective analysis of 8,533 heparin-induced extra-corporeal LDL precipitation apheresis treatments (HELP), we evaluated the efficacy of LDL reduction, the rate of adverse events, and the progression of atherosclerosis.

Methods Between July 1992 and April 2009, we performed 8,533 HELP apheresis therapies in patients with familial hypercholesterolemia $(\mathrm{FH})$. Inclusion criteria were FH with insufficient lipidological status under optimal drug therapy and diet, and at least 50 HELP therapies. Left ventricular function and valvular status was checked prior to the first apheresis therapy and at the end of the individual HELP program. Blood samples were taken directly before and after each therapy. Blood count, electrolytes, total cholesterol, LDL-C, high density lipoprotein (HDL-C), triglycerides, lipoprotein (a) (Lp(a)), and fibrinogen were measured. Adverse events were documented weekly.

Results We evaluated 27 patients (19 men) with FH (age $49.2 \pm 12.5$ years (range 10-67 years)). The number of HELP treatments once weekly was between 50 and 790 applications. Mean follow-up time was $7.0 \pm 5.2$ years (range 1.3-16.6 years).

Prior to the individual apheresis program, $44.4 \%$ of the patients had a three vessel disease (VD; 25.9\% two VD, $25.9 \%$ one VD) and $7.4 \%$ had a peripheral arterial occlusive disease. During the time of HELP treatment, none of the
\end{abstract}

F. van Buuren $(\triangle) \cdot$ S. Kreickmann · D. Horstkotte

T. Kottmann $\cdot$ K. P. Mellwig

Dept. of Cardiology, Heart and Diabetes Center North

Rhine-Westphalia, Ruhr-University of Bochum,

Georgstr. 11, 32545 Bad Oeynhausen, Germany

e-mail: fvbuuren@hdz-nrw.de patients had a myocardial infarction; 3.7\% had one percutaneous coronary intervention (PCI), 11.1\% two PCI, 14.8\% three PCI, $11.1 \% \geq$ four PCI.

The patients received 1.2 \pm 1.6 (range 0-5) PCI during follow-up time. Adverse events directly associated with HELP therapy were very rare $(<3 \%)$. Mean elimination of LDL-C was 63.49 $\pm 7.1 \%$.

Discussion The HELP apheresis therapy was well accepted by the patients in our programs. Adverse events during HELP apheresis were rare. This data is in line with the experiences published by other authors who reported an adverse event rate of $3.6 \%$ in adults. The LDL-HDL ratio, one of the strongest predictors of premature CHD events, improved significantly during the apheresis program.

Conclusion HELP is a safe, comfortable, and highly effective treatment in which adverse events are rare. It can reduce the burden of atherosclerosis, with no myocardial infarction and a low coronary intervention rate in our patients.

Keywords LDL-apheresis · Familial hypercholesterolemia · HELP therapy - Complication · Safety of treatment $\cdot$ Adverse events · Efficacy

\section{Introduction}

Coronary and vascular diseases from atherosclerosis are one of the main causes of death in developed nations [1]. Without the lipid-lowering therapy, as a result of myocardial infarction or sudden cardiac death, $50 \%$ of men with heterozygous familial hypercholesterolemia $(\mathrm{FH})$ die before the age of 50 and $25 \%$ of women before the age of 60 [2].

High plasma levels of low density lipoproteins (LDL-C) and lipoprotein(a) ( $\mathrm{Lp}(\mathrm{a}))$ are associated with an increased risk of developing atherosclerosis. The pathological lipid 
profiles not only advance significant stenosis in the epicardial coronary arteries, but also cause poor microcirculation and induce systemic circulatory disturbances, such as myocardial ischemia, peripheral artery disease, and stroke [3]. FH is the most commonly occurring congenital metabolic disorder in most populations ( 1 in 300-500 people), underlining the socioeconomic and also medical relevance of apheresis therapy. Heterozygous FH patients are typically in the range of $350-550 \mathrm{mg} / \mathrm{dl}$ total cholesterol [4].

Pharmacological and lifestyle interventions are the first choices to improve lipid profiles. However, some patients with homozygous or severe heterozygous $\mathrm{FH}$ cannot be treated sufficiently by drug therapy and diet and they require additional extracorporeal treatment [5]. LDL-C apheresis is a last treatment option for hypercholesterolemic patients resistant to conservative lipid-lowering therapy [6-9].

There are different methods of eliminating LDL-C by lipid apheresis. They are mainly based on adsorption (DALI = Direct Adsorption of Lipoproteins (Fresenius, St. Wendel, Germany); IMAL = Immuno Adsorption of Lipoproteins (Baxter, Munich, Germany); and DSA=Dextran Sulphate Adsorption (Kaneka, Osaka, Japan)). Another method focuses on Membrane Differential Filtration (MDF; Diamed, Cologne, Germany). The method of Heparin-induced Extracorporeal LDL Precipitation (HELP; Braun, Melsungen, Germany) is also well accepted and the chosen method in the presented study [2]. All methods are widely used and are supposed to provide a sufficient reduction in LDL cholesterol levels. Some authors describe a decreased susceptibility of LDL-C to oxidation after apheresis, underlining the relevance of this treatment in patients with severe FH $[10,11]$.

The suggested efficacy targets for apheresis in heterozygote $\mathrm{FH}$ patients are a reduction of at least $60 \%$ from baseline LDL-C levels [12]. In addition to its efficacy, the convenience of the method is also an important factor for keeping the patients' motivation high to join, and remain in, the apheresis programs.

Potential adverse events of extracorporeal lipid apheresis are hypotension, nausea and vomiting, flushing, headache, allergic reactions, complaints associated with anticoagulation, and hemolysis [13]. The incidence of these adverse events is usually below $5 \%$. Local problems like posttreatment venous bleeding and infections can also be seen in a few cases [5]. On the other hand, rapid relief from anginal episodes in patients with coronary heart disease (CHD) is just one positive clinical aspect frequently seen in individuals undergoing regular apheresis once weekly [14].

Based on the data obtained in our hospital, we present in this paper the results of a retrospective analysis of our HELP apheresis program with a special focus on efficacy of LDL-C reduction and potential adverse events. In addition, left ventricular function and progression of atherosclerosis were also studied.

\section{Subjects and methods}

Subjects

Between July 1992 and April 2009, we performed 8,533 HELP apheresis therapies in patients with FH. Data were analyzed retrospectively.

Inclusion criteria were FH with insufficient lipidological status under optimal drug therapy and diet and at least 50 HELP therapies. The cardiovascular risk factors (nicotine, arterial hypertension, diabetes mellitus, FH) and cardiovascular interventional history prior to the first HELP application were evaluated. The left ventricular function and valvular status were checked prior to the first apheresis therapy and at the end of the individual apheresis program. Conventional echocardiography was performed according to the ASE guidelines (GE Vingmed Seven) [15].

Blood samples were taken directly before and after each apheresis therapy. Blood count, electrolytes, total cholesterol, LDL-C, high density lipoprotein (HDL-C), triglycerides, $L p(a)$, and fibrinogen were measured. Lipidological analysis was performed using the Architect ci8200 system (Abbott). Cholesterol was measured by enzymatic assay. Fibrinogen was measured using the Sysmax CA 7000 (Siemens). From this data, we calculated the total reduction after apheresis therapy expressed in $\%$.

Until 1998, there were other reference values for $L p(a)$ (800 U/lals). This reference value has since been changed to $30 \mathrm{mg} / \mathrm{dl}$. Hence, we set both cut-off values as $100 \%$ and gave the corresponding data in $\%$ of reference value. For the evaluation of the cholesterol levels over time, the data derived before and after HELP therapy was summed up in quarters, representing 12 HELP applications. The coronary status and echocardiographic parameters were documented by an Access database.

\section{HELP therapy}

HELP works on the principle of specific precipitation of LDL-C, Lp(a), and fibrinogen at an acidic $\mathrm{pH}$ in the presence of heparin. The patient's blood is separated from the corpuscular blood elements. The plasma is then mixed with a sodium acetate buffer ( $\mathrm{pH} 4.85)$ and heparin. These heparin-protein complexes (consisting of LDL-C, Lp(a), and fibrinogen) precipitate at a reaction $\mathrm{pH}$ of 5.12 and are retained by a polycarbonate filter [16, 17]. Afterwards, the excess heparin is absorbed and the physiological plasma $\mathrm{pH}$ is restored by ultrafiltration before the plasma, along with the corpuscular elements, is returned to the patient. 
Statistical analyses

All data were documented in an Excel File (Version 2007). For the analysis and to generate the figures, the mean values of quarters were used. Plausibility tests were performed.

The statistical analyses were carried out using SPSS for Windows, version 17.0 (SPSS Inc., U.S.A.). Standard deviation was chosen as measure of dispersion. Results are given as mean values $\pm \mathrm{SD}$.

Regarding their normal distribution, the continuous variables were tested using the Shapiro-Wilk test. While some of the tested variables did not feature any normal distribution $(p<0.05)$, a normal distribution could be calculated for other variables $(\mathrm{p} \geq 0.05)$. Thus, for the comparison of the samples, tests for normally distributed samples and nonparametric tests for non-normally distributed samples were used.

For the comparison of two independent, normally distributed samples, the t-test was applied. In cases of abnormal distribution, the Wilcoxon test was used. A p-value of $<0.05$ (alpha level) was assumed as statistically significant. Clinical data derived from echocardiogram and angiogram were evaluated with the help of an Access database (Version 2007).

\section{Results}

We evaluated 27 patients (19 men) with FH (age 49.2 \pm 12.5 years (range 10-67 years)). Height was $172 \pm 9 \mathrm{~cm}$, weight $73.9 \pm 15.8 \mathrm{~kg}$, (BMI $\left.24.7 \pm 3.6 \mathrm{~kg} / \mathrm{m}^{2}\right)$. Other cardiovascular risk factors were arterial hypertension (63\%), former smoking (37\%), diabetes mellitus (14.8\%), and current smoking $7.4 \%)$.

The number of HELP treatments once weekly was between 50 and 790 applications (Table 1). The mean number of HELP treatments was $309 \pm 246$. More than $50 \%$ of the patients had $>230$ HELP therapies. Mean follow-up time was $7.0 \pm 5.2$ years (range $1.3-16.6$ years).

Twenty six patients had a CHD when starting HELP therapy, one patient with no initial CHD suffered from a minor stroke and developed CHD afterwards. The mean age of the patients at the time of CAD diagnosis was $43.8 \pm 10.6$ years (range between 13 and 65 years). When starting the individual apheresis program, $44.4 \%$ had a three vessel disease (VD; 25.9\% two VD, 25.9\% one VD); 7.4\% had an additional peripheral arterial occlusive disease treated by angioplasty and $44 \%$ had coronary bypass graft operation (37\% myocardial infarction) before starting the apheresis program.

During the time of HELP treatment, none of the patients had a myocardial infarction. Sixteen patients $(59.3 \%)$ had stable CHD and there was no need for percutaneous coro-
Table 1 Start and end of followup time in 27 patients receiving HELP therapy

\begin{tabular}{|c|c|c|c|}
\hline Patient No. & Start of HELP & End of HELP & Number of procedures \\
\hline 1 & 16.12 .1998 & 12.01 .2004 & 226 \\
\hline 2 & 25.04 .2001 & 18.02.2004 & 68 \\
\hline 3 & 16.10 .1992 & 20.09 .1996 & 185 \\
\hline 4 & 02.02 .1996 & 08.04.2009 & 631 \\
\hline 5 & 14.03.2007 & 07.04.2009 & 101 \\
\hline 6 & 09.11 .1994 & 26.05.1998 & 50 \\
\hline 7 & 25.04 .2007 & 15.04 .2009 & 93 \\
\hline 8 & 11.11.2005 & 03.04.2009 & 111 \\
\hline 9 & 11.12 .2003 & 26.10 .2007 & 67 \\
\hline 10 & 03.08.1999 & 09.04.2009 & 419 \\
\hline 11 & 30.06 .1992 & 15.04 .2009 & 758 \\
\hline 12 & 08.07 .1992 & 14.04 .2009 & 751 \\
\hline 13 & 10.03 .1994 & 04.05 .2005 & 527 \\
\hline 14 & 26.04 .2007 & 15.04.2009 & 91 \\
\hline 15 & 11.02 .2003 & 15.04.2009 & 291 \\
\hline 16 & 17.01.2007 & 14.04.2009 & 116 \\
\hline 17 & 21.04 .1994 & 15.04.2009 & 686 \\
\hline 18 & 05.05 .1994 & 16.01 .2004 & 472 \\
\hline 19 & 11.01 .1993 & 14.04.2009 & 790 \\
\hline 20 & 03.03 .2005 & 14.04.2009 & 56 \\
\hline 21 & 23.05 .2005 & 06.04 .2009 & 169 \\
\hline 22 & 17.05.1999 & 29.03.2004 & 230 \\
\hline 23 & 15.04 .1996 & 08.04 .2009 & 437 \\
\hline 24 & 16.09 .2003 & 08.04.2009 & 249 \\
\hline 25 & 16.04 .1998 & 17.01.2001 & 65 \\
\hline 26 & 05.05 .1998 & 09.04.2009 & 463 \\
\hline 27 & 13.02 .2003 & 03.04.2009 & 253 \\
\hline
\end{tabular}

nary intervention (PCI). One patient $(3.7 \%)$ had 1 , three patients $(11.1 \%)$ had 2, four patients $(14.8 \%)$ had 3 and three patients $(11.1 \%)$ had $\geq 4$ coronary angioplasties. The patients received $1.2 \pm 1.6$ (range $0-5$ ) PCI during follow-up time.

Prior to initial apheresis treatment, echocardiography revealed a good left ventricular function with an ejection fraction (EF) $>55 \%$ in $85.2 \%$ (EF 40-50: 3.7\%; EF 30-40: $3.7 \%, \mathrm{EF}<30: 7.4 \%)$. On follow-up, 23 patients $(85.2 \%)$ presented with the same EF, 2 patients $(7.4 \%)$ showed a worsened EF, 2 patients (7.4\%) with an improved EF.

Prior to initial application of HELP therapy, the cholesterol levels were significantly increased in all individuals despite drug therapy. Total cholesterol was $308 \pm 96 \mathrm{mg} /$ dl (LDL-C $223 \pm 96 \mathrm{mg} / \mathrm{dl}$, HDL-C $52 \pm 16 \mathrm{mg} / \mathrm{dl}$, triglycerides $216 \pm 210 \mathrm{mg} / \mathrm{dl}$, fibrinogen $307 \pm 78 \mathrm{mg} / \mathrm{dl}$, Lp(a) $172.9 \pm 199.8 \%$ ).

Figure 1 shows the lipid and fibrinogen profile during the time of treatment. Figure 2 gives an overview of the degree of LDL-C reduction after each treatment. The mean elimination of LDL-C (measurement immediately before and 
Fig. 1 Lipid and fibrinogen profile during the time of treatment. The blue line indicates concentrations before the initial treatment
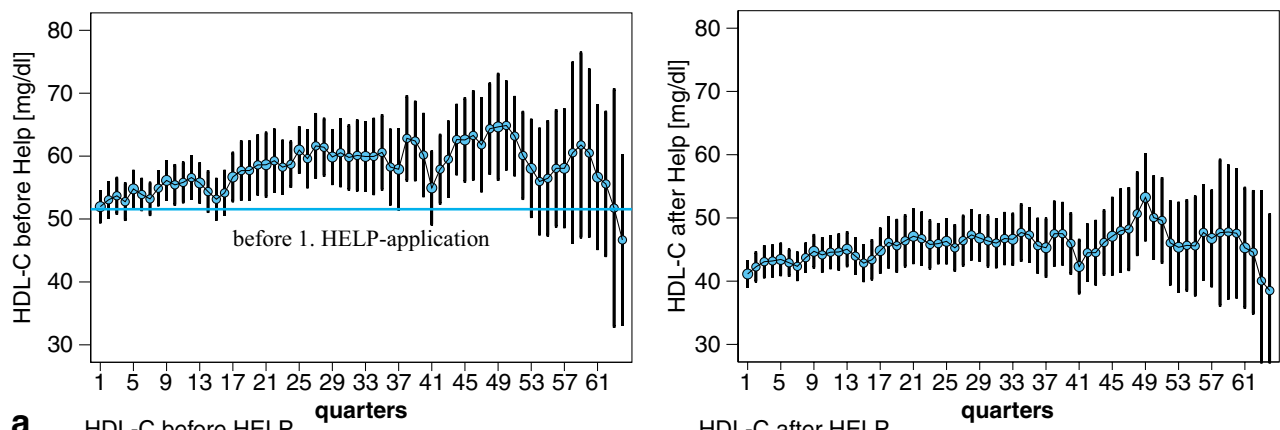

a
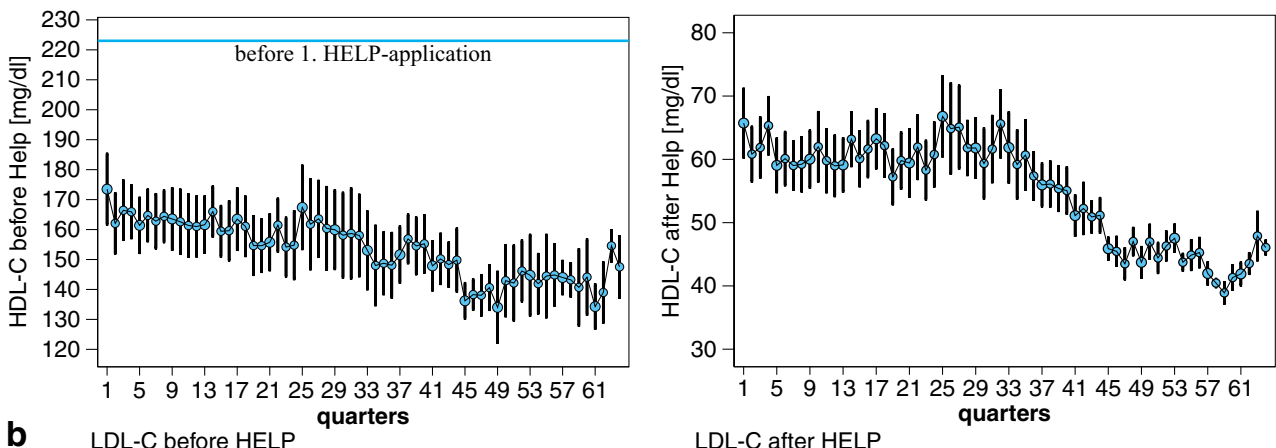

LDL-C after HELP

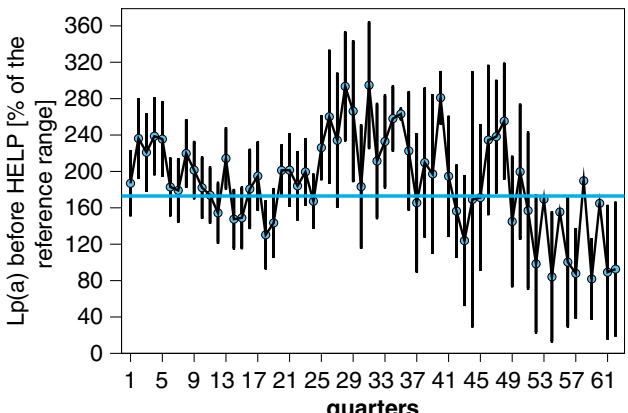

C

Lp(a) before HELP

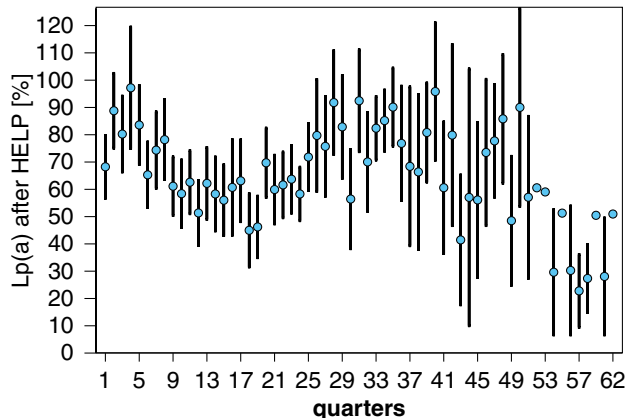

LP(a) after HELP

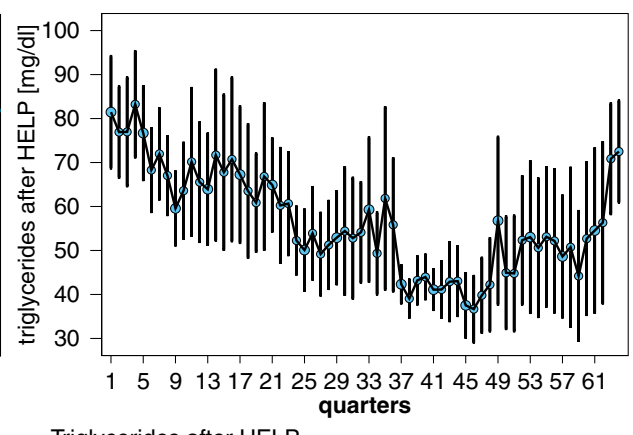

after each HELP therapy) was $63.49 \pm 7.1 \%$ (HDL $+15.4 \%$, total cholesterol $-59.1 \%$, triglycerides $-62.3 \%$, fibrinogen $-68.6 \%, \mathrm{Lp}(\mathrm{a})-60.6 \%)$.

All patients were on CSE inhibitor before the initial HELP therapy (simvastatin 51.9\%; atorvastatin 37\%, pravastatin $3.7 \%$, one patient other); $11 \%$ had supplement fibrates, $3.7 \%$ nicotin acid.
Complications

During the follow-up time, none of the patients had severe arrhythmia (e.g. ventricular tachycardia), major bleeding, or severe allergic reaction during HELP therapy. Two patients were operated for coronary artery bypass graft. Two patients died (one from CHD, another from a non-cardiovascular disease). One patient developed sepsis because of a leg 
Fig. 2 a Treatment efficacy of HELP therapy and, b Ratio of LDL-HDL over time

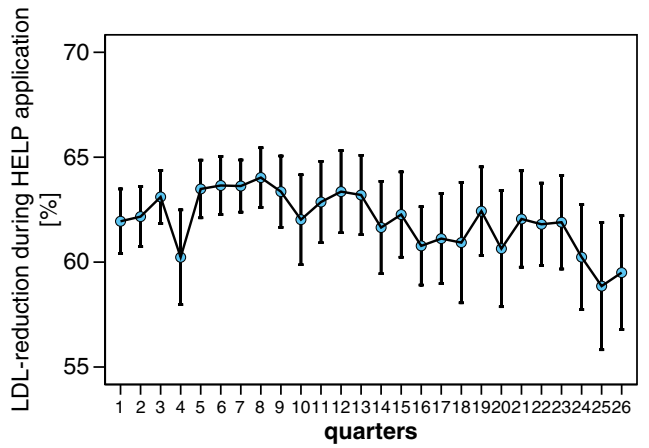

a Reduction of LDL-C after each HELP therapy in \%

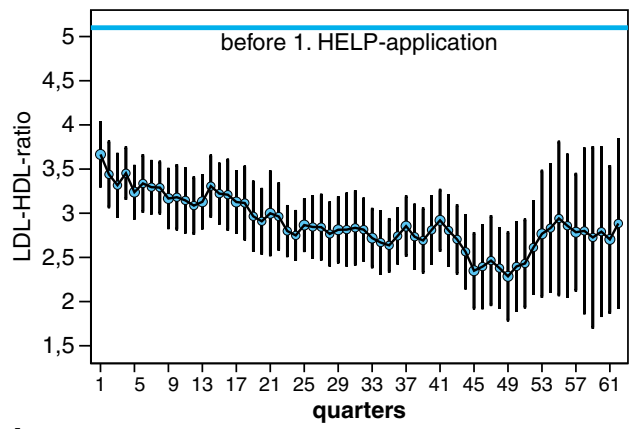

b LDL / HDL ratio over time phlegmone, another received an implantable cardioverterdefibrillator for secondary prophylaxis.

Minor adverse events directly associated with HELP therapy, such as nausea, hypotension, headache, abdominal or chest pain, were seen in a small percentage of treatments $(<3 \%)$. During follow-up, we saw one local major infection as a result of venous puncture.

\section{Discussion}

Recently published data state that LDL-C apheresis is an approved medical therapy for patients who have not reached the treatment target after 6 months of maximum tolerated drug therapy or who have ongoing symptomatic disease [4]. In the presented study, HELP apheresis could lower LDL-C by more than $60 \%$, confirming the efficacy of this treatment.

Besides the positive effects on decreased susceptibility of LDL-C to oxidation, it is also described in the literature that HELP apheresis can improve myocardial blood flow at rest and under stress in patients with CHD [18]. Mellwig $[19,20]$ demonstrated by positron emission tomography (PET) that even a single HELP apheresis therapy can improve coronary vasodilatation by $30 \%$ within $24 \mathrm{~h}$. This is concomitant with an improved endothelial function with increasing antiischemic, antithrombotic, and antiproliferative effects. Hence, the low rate of cardiovascular events and the stable EF in more than $90 \%$ of the patients (e.g. there was no further myocardial infarction during the time of the individual HELP apheresis program) in the presented study can be better understood. Also the frequency of coronary intervention can be traced back to improved microcirculatory status and the reduced susceptibility of LDL to oxidation. However, this finding might be influenced by the very high frequency of "patient/doctor contacts" and the resulting intensive monitoring of complaints. To provide an optimal treatment for the patients, we work in our center with a team of two doctors and five dedicated apheresis nurses with excellent skills.
In the last few years, there have been several reports on the additional use of apheresis in patients with acute hearing loss and peripheral arterial disease. [21, 22]. The important mechanism of the positive effects of apheresis is supposed to be a reduced viscosity of the blood because of the reduction in LDL-C and fibrinogen. Since fibrinogen, in particular, is a large glycoprotein that defines rheological characteristics of blood and its potential of aggregation of erythrocytes, thrombocytes, and leucocytes, it is thought to be a main factor for reduced microcirculation [21]. In our study, we could also, in addition to LDL-C, achieve a very effective elimination of fibrinogen and $\mathrm{Lp}(\mathrm{a})$, suggesting that HELP therapy might influence the clinical outcome of patients with acute hearing loss or peripheral artery disease to a high extent.

Lipoprotein (a) is known as an independent risk factor for premature CHD that can only be treated sufficiently by lipid apheresis therapy [23]. However, only scant data about the role of $\mathrm{Lp}(\mathrm{a})$ and the risk of major cardiovascular events are available. We could show a relevant reduction in $\mathrm{Lp}(\mathrm{a})$ of more than $60 \%$, but we cannot comment on the influence of this finding on the progress of atherosclerosis since all individuals also suffer from FH. Hence, further trials are needed to find definite answers concerning the role of elevated $\mathrm{Lp}(\mathrm{a})$ levels.

Also, HDL-C plays an important role in the genesis of atherosclerosis. An inverse association of HDL-C levels and rates of CHD incidence and mortality was observed in prospected studies in the 1970s [24]. It was reported that each $1 \mathrm{mg} / \mathrm{dl}$ increment in baseline HDL-C was associated with a $5.5 \%$ decrement in risk of definite CHD death or myocardial infarction. The exact mechanisms by which HDL-C is atheroprotective are multifactorial [25]. The most recognized effects are to protect LDL-C from oxidation, the initiation of mobilization of cholesterol from the arterial wall and the decrease in endothelial cell adhesion molecules. HDL-C increased slightly during the time of treatment in our study, which is in contrast to findings of other authors who describe a chance of HDL-C elimination of between 5 
and $17 \%$ in patients receiving HELP therapy [26]. However, HDL-C reduction in our collective was not significant.

The LDL-HDL ratio is one of the strongest predictors of premature CHD events. It could be shown that in patients with a ratio of $>5.0$ in combination with triglyceride concentrations of $>200 \mathrm{mg} / \mathrm{dl}$ the risk of coronary events compared to those with lower LDL/HDL ratio and triglycerides $<200 \mathrm{mg} / \mathrm{dl}$ is four times higher [27]. In our patients, the LDL/HDL ratio improved significantly during the apheresis program and reached $2.7 \pm 0.1$ (initial data before HELP therapy $5.1 \pm 3.8$ (Fig. 2)). The triglyceride concentration could be reduced by $63 \%$ directly after apheresis.

HELP apheresis therapy was very well accepted by the patients in our programs. This is most likely explained by the very low frequency of adverse events of $<3 \%$ during HELP apheresis. This data is similar to experiences published by other authors who reported $3.6 \%$ in adults and 3\% in children $[28,29]$. In particular, major problems like severe arrhythmia or inflammatory diseases causing further in-hospital treatment did not occur.

\section{Conclusion}

HELP apheresis is an accepted and highly effective therapeutic option in patients with $\mathrm{FH}$ who cannot be treated sufficiently by drug therapy and lifestyle intervention. It is a safe and comfortable treatment in which adverse events are rare. HELP is suitable for reducing LDL-C concentrations by more than $60 \%$ and might help to reduce the burden of atherosclerosis as there was no myocardial infarction and a low coronary intervention rate in our patients. Apheresis also seems to be a therapeutic option in noncardiac diseases such as acute hearing loss and peripheral arterial disease, but this has to be confirmed by further trials.

\section{Acknowledgments This article is part of a supplement sponsored by an unrestricted educational grant from B. Braun and Fresenius Medical Care.}

Conflict of interest The author declares that there are no conflicts of interest.

Open Access This article is distributed under the terms of the Creative Commons Attribution License which permits any use, distribution, and reproduction in any medium, provided the original author(s) and the source are credited.

\section{Literature}

1. Lloyd-Jones DM, Larson MG, Beiser A, Levy D (1999) Lifetime risk of developing coronary heart disease. Lancet 353:89-92

2. Krebs A, Krebs K, Keller F (2004) Retrospective comparison of 5 different methods for long-term LDL-apheresis in 20 patients between 1986 and 2001. Int J Artif Organs 27:137-148
3. Koga N (2001) Effects of low-density lipoprotein apheresis on coronary and carotid atherosclerosis and diabetic scleredema in patients with severe hypercholesterolemia. Ther Apher 5:244-251

4. Goldberg AC, Hopkins PN, Toth PP et al (2011) Familial hypercholesterolemia: screening, diagnosis and management of pediatric and adult patients: clinical guidance from the National Lipid Association Expert Panel on Familial Hypercholesterolemia. J Clin Lipidol 5:S1-8

5. Klingel R, Mausfeld P, Fassbender C, Goehlen B (2004) Lipidfiltration - safe and effective methodology to perform lipid apheresis. Transfus Apher Sci 30:245-254

6. Bosch T, Wendler T (2004) State of the art of low-density lipoprotein apheresis in the year 2003. Ther Apher Dial 8:76-79

7. Schettler V, Jaeger BR, Klingel R (2009) The German lipid apheresis registry-remaining to be established. Atheroscler Suppl 10:59-61

8. Thompson GR (2010) Lipoprotein apheresis. Curr Opin Lipidol 21:487-491

9. Thompsen J, Thompson PD (2006) A systematic review of LDL apheresis in the treatment of cardiovascular disease. Atherosclerosis 189:31-38

10. Donner MG, Parhofer KG, Richter WO, Schwandt P (1999) Lowdensity lipoprotein (LDL) oxidizability before and after LDL apheresis. Metabolism 48:881-886

11. Pulawski E, Mellwig KP, Horstkotte D (2003) H.E.L.P. apheresis and oxidative stress. Z Kardiol 92(Suppl 3):III38-41

12. Thompson GR, Barbir M, Davies D, Dobral P, Gesinde M, Livingston M, Mandry P, Marais AD, Matthews S, Neuwirth C, Pottle A, le Roux C, Scullard D, Tyler C, Watkins S (2010) Efficacy criteria and cholesterol targets for LDL apheresis. Athero sclerosis 208:317-321

13. Thompson GR, Catapano A, Saheb S, Atassi-Dumont M, Barbir M, Eriksson M, Paulweber B, Sijbrands E, Stalenhoef AF, Parhofer KG (2010) Severe hypercholesterolaemia: therapeutic goals and eligibility criteria for LDL apheresis in Europe. Curr Opin Lipidol 21;492-498

14. Schuff-Werner P, Holdt B (2002) Selective hemapheresis, an effective new approach in the therapeutic management of disorders associated with rheological impairment: mode of action and possible clinical indications. Artif Organs 26:117-123

15. Hunt SA, Fuster V, Jacobs AK, Gibbons RJ, Russell RO (2003) American College of Cardiology; American Heart Association; American Society of Echocardiography. American College of Cardiology; American Heart Association; American Society of Echocardiography. ACC/AHA/ASE 2003 guideline update for the clinical application of echocardiography: summary article: a report of the American College of Cardiology/American Heart Association Task Force on Practice Guidelines. Circulation 108:1146-1162

16. Mellwig KP (2003) Heparin-induced extracorporeal low-density lipoprotein precipitation. Ther Apher Dial 7:365-369

17. Horstkotte D, Mellwig KP (2003) Heparin-induced extracorporeal LDL precipitation (H.E.L.P.). Z Kardiol 92(Suppl 3):III1-5

18. Jaeger BR (2001) Evidence for maximal treatment of atherosclerosis: drastic reduction of cholesterol and fibrinogen restores vascular homeostasis. Ther Apher 5:207-211

19. Mellwig KP, Baller D, Gleichmann U, Moll D, Betker S, Weise R, Notohamiprodjo G (1998) Improvement of coronary vasodilatation capacity through single LDL apheresis. Atherosclerosis 139:173-178

20. Mellwig KP, Baller D, Schmidt HK, van Buuren F, Wielepp JP, Burchert W, Horstkotte D (2003) Myocardial perfusion under H.E.L.P.-apheresis. Objectification by PET. Z Kardiol 92(Suppl 3):III30-7 
21. Suckfuell M, Hearing Loss Study Group (2002) Fibrinogen and LDL apheresis in treatment of sudden hearing loss: a randomised multicentre trial. Lancet 360:1811-1817

22. Kamimura M, Matsuo M, Miyahara T, Kimura K, Matsumoto K, Nakaya T, Abe T, Akizawa T (2002) Improvements in artery occlusion by low-density lipoprotein apheresis in a patient with peripheral arterial disease. Ther Apher 6:467-470

23. Kassner U, Vogt A, Rosada A, Barz F, Giannakidou-Jordan E, Berthold HK, Steinhagen-Thiessen E (2009) Designing a study to evaluate the effect of apheresis in patients with elevated lipoprotein(a). Atheroscler Suppl 10:85-88

24. Gordon DJ, Knoke J, Probstfield JL, Superko R, Tyroler HA (1986) High-density lipoprotein cholesterol and coronary heart disease in hypercholesterolemic men: the lipid research clinics coronary primary prevention trial. Circulation 74:1217-1225

25. Moriarty PM, Gibson CA (2005) Association between hematological parameters and high-density lipoprotein cholesterol. Curr Opin Cardiol 4:318-323
26. Thompson GR (2008) Recommendations for the use of LDL apheresis. Atherosclerosis 198:247-255

27. Panagiotakos DB, Pitsavos C, Skoumas J, Chrysohoou C, Toutouza M, Stefanadis CI, Toutouzas PK (2003) Importance of $\mathrm{LDL} / \mathrm{HDL}$ cholesterol ratio as a predictor for coronary heart disease events in patients with heterozygous familial hypercholesterolemia: a 15-year followup (1987-2002). Curr Med Res Opin 19:89-94

28. Gordon BR, Kelsey SF, Dau PC, Gotto AM Jr, Graham K, Illingworth DR, Isaacsohn J, Jones PH, Leitman SF, Saal SD, Stein EA, Stern TN, Troendle A, Zwiener RJ (1998) Long-term effects of low-density lipoprotein apheresis using an automated dextran sulfate cellulose adsorption system. Liposorber Study Group. Am J Cardiol 81:407-411

29. Hudgins LC, Kleinman B, Scheuer A, White S, Gordon BR (2008) Long-term safety and efficacy of low-density lipoprotein apheresis in childhood for homozygous familial hypercholesterolemia. Am J Cardiol 102:1199-1204 